Received: 13 March 2018

Accepted: 7 August 2018

Published online: 26 October 2018

\section{NADPH oxidase NOX4 is a glycolytic regulator through mROS- HIF1 $\alpha$ axis in thyroid carcinomas}

Ping Tang ${ }^{1}$, Hao Dang ${ }^{2}$, Jie Huang ${ }^{3}$, Tao Xu ${ }^{1}$, Ping Yuan ${ }^{1}$, Jun Hu \& Jian-feng Sheng $^{1}$

The function of the NAD(P)H oxidases (NOXs) family member NOX4 is to generate reactive oxygen species (ROS), however, the molecular function of NOX4 has not been fully studied and waiting to be clarified. To elucidate the function of endogenous Nox 4 in human thyroid carcinomas, papillomatosis thyroid cancer cells were used to study the cell growth by knocking down the expression of NOX4 and knocking out its functional partner p22phox/CYBA. As a result, the increasement of mitochondrial ROS(mROS) was abolished due to both knockdown of NOX4 and p22phox knockout in hypoxia, which destabilized HIF1 $\alpha$ decreasing glycolysis and retarded cell growth. These data suggests that Nox4 is potent oncotarget due to its role in regulating glycolysis through mROS-HIF1 $\alpha$ pathway, thereby mediating proliferation in thyroid carcinomas.

Papillary thyroid cancer (PTC) is the most common histologic type of human thyroid carcinoma that continues to be the most rapidly increasing cancer ${ }^{1}$. Although partially due to overdiagnosis because of increased use of advanced imaging techniques, occasionally they dedifferentiate into more aggressive and lethal thyroid cancers ${ }^{2}$. Therefore, investigating the underlying molecular mechanisms of PTC can provide promising biomarkers and therapeutic targets for early diagnosis and treatment, thus improving prognosis and survival quality of patients, especially those with aggressive tumor behavior and adverse outcomes.

Previously, ROS was detected at the apical surface of thyrocytes, indicating a relatively high level of this oxidizing agent in the thyroid gland ${ }^{3,4}$. More recently, the observation that somatic mutations are present in higher levels in the rat thyroid gland has further confirmed that the thyrocyte is under oxidative stress ${ }^{5}$. Unlike other oxidoreductases that generate ROS only as by-products along their specific catalytic pathways, NOXs family are 'professional' producers of ROS, as their primary function is to generate these molecules ${ }^{6}$. Among the NOXs family NOX4 is expressed at a high level in human thyroid tumours and is controlled at the transcriptional level by thyroid Stimulating Hormone(TSH) unlike dual oxidases(DUOXs) $)^{7,8}$. Heterodimerization of NOX4 with the p22phox is able to increase ROS production ${ }^{9}$. However, the source of ROS, possibly contributing to various disorders associated with enhanced proliferation in PTC, involved in NOX4 has only recently begun to be clarified.

The metabolism of malignant tumors can be explained with Warburg effect, a metabolic shift from oxidative phosphorylation (OXPHOS) to glycolysis in tumor cells ${ }^{10}$. Hypoxic microenviroment induces the shift and stabilizes hypoxia-inducible transcription factors(HIFs), which associated with the regulation of glycolysis and the shift to a suppression of oxidative metabolism ${ }^{11}$. However, its stabilization is required for the ROS production, which happen to depend directly on NOX4 expression in PTC.

In the present article, we describe the role of NOX4 play a part not only in PTC proliferation but also in cellular metabolism in hypoxic PTC. The aim of the study was to analyze the sources of mROS in hypoxia sustained by NOX 4 and to explore the contribution of glycolysis induced by NOX4/p22phox on PTC proliferation and metabolism.

${ }^{1}$ Otorhinolaryngology Head and Neck Surgery, The Third Hospital of Mianyang(Sichuan mental health center), No. 190 The East Jiannan Road, Mianyang, 621000, Sichuan, People's Republic of China. ${ }^{2}$ Department of Clinical Laboratory, The Third Hospital of Mianyang(Sichuan mental health center), No. 190 The East Jiannan Road, Mianyang, 621000, Sichuan, People's Republic of China. ${ }^{3}$ Yunnan Jiehui Biotech Ltd., the KunMing economic and Technological Development Zone, No. 9 The Daxi Road, 650215, KunMing, Yunnan, People's Republic of China. Ping Tang and Hao Dang contributed equally. Correspondence and requests for materials should be addressed to J.-f.S. (email: ShengJianfeng0901@163.com) 
A

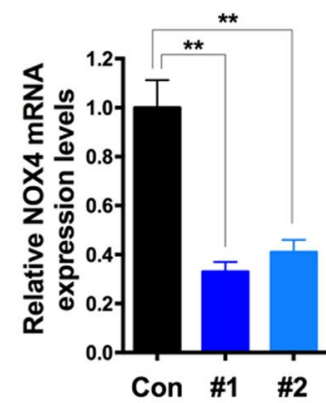

B

ShRNA

NoX4

Actin

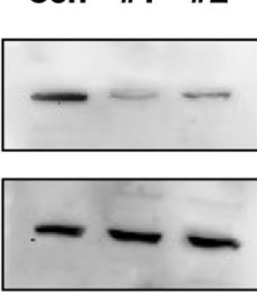

C

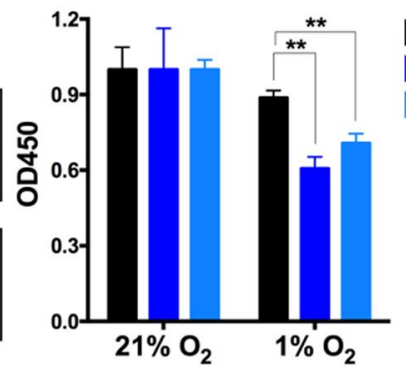

shControl

shNOX4\#1

shNOX4\#2

D

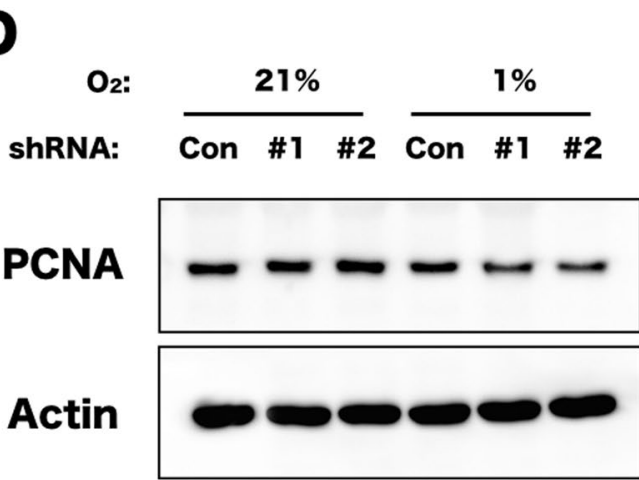

E

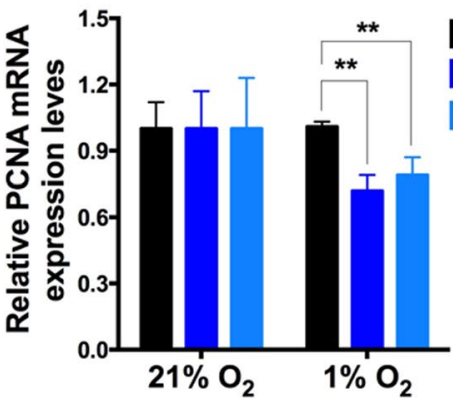

shControl

shNOX4\#1

shNOX4\#2

$\mathbf{F}$
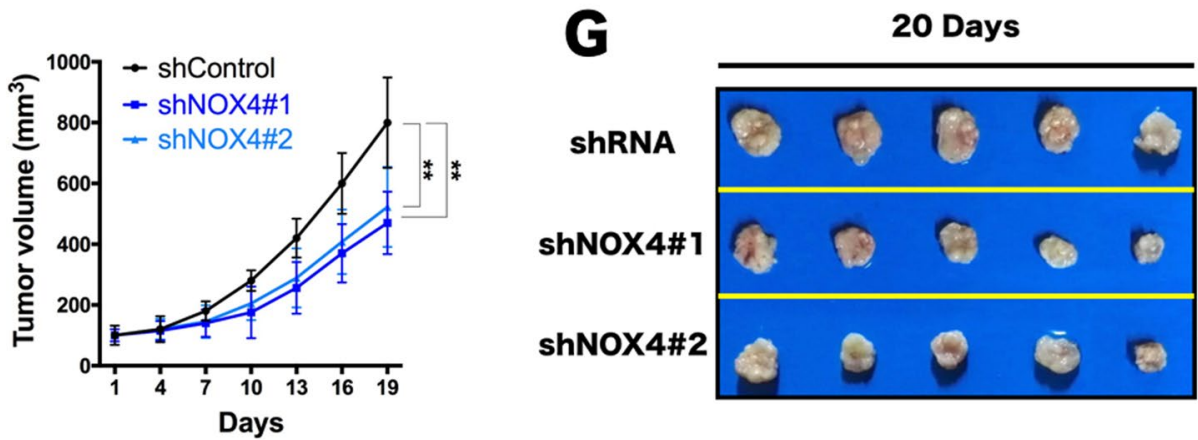

Figure 1. NOX4 Knockdown results in inhibition of TPC-1 Proliferation. (A,B) Transcriptional expression of NOX4 in TPC-1 cells after 48 hours treated with lentiviral transduction particles targeting NOX4 mRNA (A). Protein expression level of NOX4 after 72 hours treated with lentiviral transduction particles targeting NOX4 mRNA (B). Con for shNOX4 control lentivirus, \#1 for shNOX4\#1 lentivirus, and \#2 for shNOX4\#2 lentivirus. $* * \mathrm{P}<0.01$. (C) Viability assay for TPC-1 cells expressing shControl or shRNA against NOX4 (shNOX4\#1,\#2) which were cultured in normoxia $\left(21 \% \mathrm{O}_{2}\right)$ and hypoxia $\left(1 \% \mathrm{O}_{2}\right)$ respectively for 48 hours using CCK8 assay $(\mathrm{n}=8)$. $* * \mathrm{P}<0.01$. (D,E) Western blot for normoxia $\left(21 \% \mathrm{O}_{2}\right)$ and hypoxia $\left(1 \% \mathrm{O}_{2}\right)$ in TPC-1 cell clones after infected with either shNOX4 control lentivirus and shNOX4\#1 and shNOX4\#2 lentivirus (D). The blots were quantified using ImageJ software $(\mathrm{n}=3)$. $* * \mathrm{P}<0.01$. (F,G) TPC-1 cells transduced with shNOX4 control or two NOX4-directed shRNAs were injected subcutaneously in the flanks of nude mice. Tumor growth was quantified with a caliper at the indicated time intervals for 20 days $(\mathbf{F})$. After the measurement, these mice were euthanized and then stripped of the subcutaneous transplantation tumor to take pictures at 20 days (G). Data were analyzed using the two-sided unpaired Student's t test. Mean \pm SEM. **p $<0.01$.

\section{Results}

TPC-1 proliferation is inhibited due to NOX4 knockdown. To investigate the role of NOX4 in the proliferation of thyroid cancer cells, two NOX4-knockdown cell stains were established by short hairpin RNA(shRNA) and NOX4 was severely interfered in the strain TPC-1 (Fig. 1A,B). Then we found that the viability of the knockdown cells using cell counting kit-8(CCK8) did not have a obvious change under common conditions (Fig. 1C). Considering the growth microenvironment of tumor cells, cells was put in the hypoxic incubator ( $1 \%$ $\mathrm{O}_{2}$ ) to mimic growth condition. Compared to control cell strain in hypoxia, the growth of shRNA targeting cells was decreased by nearly $30 \%$ (Fig. 1C), and very similar phenotypes also appeared in other two papillary thyroid cancer cell lines K1 and BCPAP (Supplementary Fig. S1). 
A

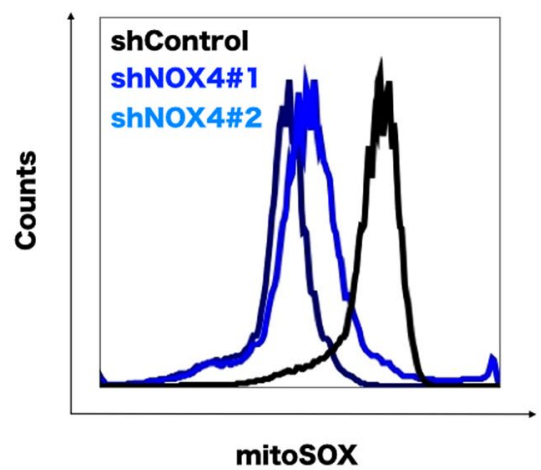

C

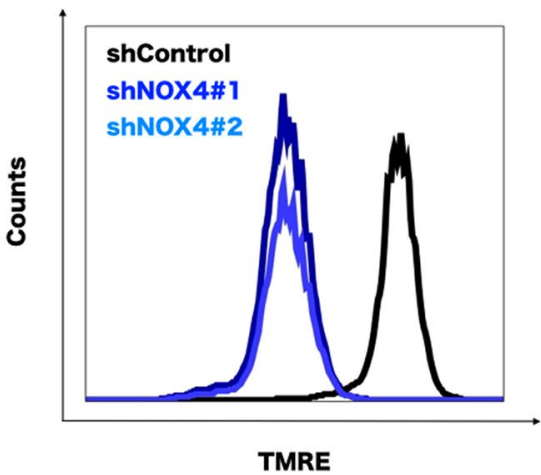

B

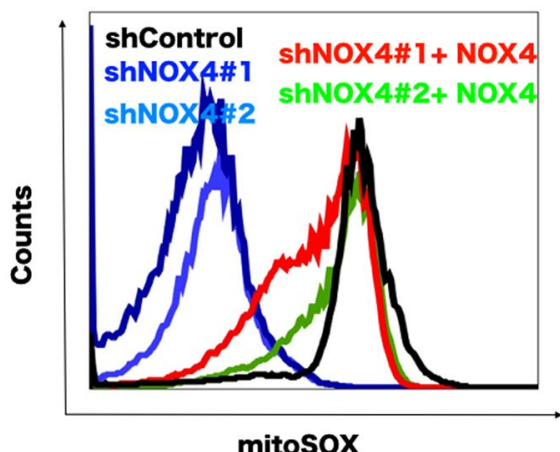

D

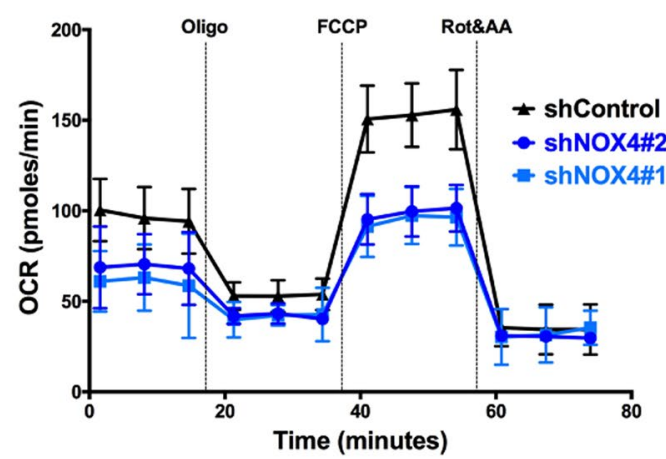

Figure 2. NOX4 is required for mitochondrial function in thyroid cancer during hypoxia. (A,B) Mitochondrial ROS level was measured with MitoSox staining by flow cytometry analysis in TPC-1 cells cultured in $1 \% \mathrm{O}_{2}$ for 48 hours (A). NOX4 transfection can rescue mROS production decreasing after cell was treated with $1 \% \mathrm{O}_{2}$ for 48 hours (B). (C) Mitochondrial membrane potential assessed by TMRE (50 nM) staining of NOX4 knockdown TPC-1 cells. (D) Whole-TPC-1 cell oxygen consumption rate (OCR) in different groups. The dotted lines indicate the time of adding oligomycin $(1 \mu \mathrm{M})$, FCCP $(1 \mu \mathrm{M})$ and rotenone and antimycin A (Rot/AA, $1 \mu \mathrm{M}$ each).

To further investigate the causes of cell proliferation decline under hypoxia, the protein immune blot after lysating cells showed that, the proliferating cell nuclear antigen (PCNA) expression level in the NOX4 knockdown cells under hypoxia was downregulated (Fig. 1D,E), highlighting the effect of NOX4 in regulating the growth of thyroid cancer cells under hypoxic microenvironment. Otherway, NOX4 knockdown cells exhibited little change when using both Casepase 3 detection and Annexin V \& PI staining after hypoxic treatment (Supplementary Fig. S2), delineating the in vitro reduction in growth observed is not due to active cell death through apoptosis.

To determine in vivo tumorigenesis, cells were implanted into athymic nude mice. After about 1 weeks, the two NOX4 knockdown cell strains gave rise to smaller tumors, which suggests that loss of NOX4 provides a necessary genetic event resulting in tumorigenesis (Fig. 1F,G). These data suggest that NOX4 is essential for thyroid tumor maintenance.

NOX4 is required for mROS level upon hypoxia. NOX4 was described to play a role in superoxide and hydrogen peroxide production, so we evaluated its contribution of increased intracellular ROS using mitochondrial indicator, mitoSOX, upon hypoxia in thyroid cancer cells and investigate the reason why thyroid cancer proliferation was decreased by NOX4 inhibition upon hypoxia in vitro. Unsurprisingly, knockdown of NOX4 led to largely lowered mROS compared to the control levels in hypoxia (Fig. 2A). Importantly, when the vector of NOX4 overexpression was transfected into NOX4 knockdown cells, the level of mROS was recovered in hypoxia (Fig. 2B). This manipulation was consistent with the previous reports about relationship of NOX4 and ROS ${ }^{12}$.

However, if the reduction in proliferation in fact due to lowered ROS, the effect that treating NOX4 knockdown cells with low doses of hydrogen peroxide $\left(\mathrm{H}_{2} \mathrm{O}_{2}\right)$ is able to rescue previous decreased proliferation in vitro should theoretically be observed. To verify this conjecture, $\mathrm{H}_{2} \mathrm{O}_{2}$ or an $\mathrm{H}_{2} \mathrm{O}_{2}$ generating enzyme, glucose oxidase, with a concentration gradient was added to the NOX4 knockdown TPC-1 cells during hypoxia. As a result, we found that either $\mathrm{H}_{2} \mathrm{O}_{2}$ or glucose oxidase with relative lower concentration can stimulate cell proliferation, but high concentration of both inhibits cell growth (Supplementary Fig. S3). The possible reason is that as a 
double-edged sword, $\mathrm{H}_{2} \mathrm{O}_{2}$ with exorbitant concentration is easy to cause oxidative damage and then counteract the effect of its stimulation.

As NOX4 is located in mitochondrial, we asked if NOX4 would affect the mitochondrial activity by staining the cells with a probe, TMRE, to monitor the membrane potential of mitochondrial. Using flow cytometry, the results showed that, the mitochondrial membrane potential was decreased when NOX4 was deleted in hypoxia, suggesting potent relevance between mitochondrial membrane potential and mROS under hypoxia in thyroid cancer (Fig. 2C). Consistent with previous reports, mitochondrial metabolism, indicated by oxygen consumption rate (OCR), was down-regulated in the basal and maximum respiration when NOX4 was knocked down relative to the control cells pretreated with hypoxia (Fig. 2D). Altogether, NOX4 reduces mitochondrial activity by decreasing oxygen consumption, mROS and mitochondrial membrane potential under hypoxia in thyroid cancer.

NOX4 maintains HIF1 $\alpha$ stability in thyroid tumor cells. HIF1, the heterodimer of HIF1a and HIF1b, is the primary driver of increased glycolysis and lactate production during hypoxia. Under conditions of low oxygen, HIF $1 \alpha$ is stabilized by mROS and promotes transcription of many genes crucial for the cellular response to hypoxia $^{13,14}$. Consequently, cells lacking HIF1 $\alpha$ fail to upregulate glycolytic enzymes and lactic acid production in response to hypoxia. Given the mROS regulation by NOX4 under hypoxia in thyroid cells, in addition that the way of HIF1 $\alpha$ stabilization is dependent on mROS, we reasoned that the mechanism by which NOX4 regulates HIF1 $\alpha$ involves mROS in thyroid cells.

To test this idea we first investigated whether NOX4 directly modulates HIF1 $\alpha$ stability under hypoxic conditions. Generally in the presence of high oxygen, HIF1 $\alpha$ was rapidly degraded and difficult to measure in cells, but HIF1 $\alpha$ was detectable under hypoxia conditions. Surprisingly, NOX4-deficient cells during hypoxia demonstrated low levels of HIF1 $\alpha$ relative to control cells (Fig. 3A). Notably, HIF1 $\alpha$ was decreased at each of the time points in cells after transfected with NOX4 knockdown vector under hypoxia, suggesting that the reason for destabilization of HIF $1 \alpha$ was due to gradually reduced NOX4 expression (Fig. 3B,C). Furthermore, the HIF $1 \alpha$ targets genes, carbonic anhydrase 9(CA9) and solute carrier family 2 member 1(SLC2A1), were significantly decreased in NOX4 knockdown cells compared to control cells during hypoxia (Fig. 3D,E).

Tumor cells exhibits metabolism characterized by high glycolysis especially when lacking of oxygen, HIF $1 \alpha$ triggers tumor cells to upregulate glycolytic enzymes and lactic acid production in response to hypoxia ${ }^{15}$. Consistent with HIF1 $\alpha$ destabilization by NOX4 knockdown under hypoxia, bioenergetics profiling of NOX4-deficient TPC-1 revealed glycolytic loss less than the control cells: Extracellular acidification rate (ECAR) decreased obviously, suggesting NOX4 plays an essential role in the maintenance of glycolysis during TPC-1 hypoxic metabolic process (Fig. 3F). Based these results, it is suggested that NOX4 is critical for glycolytic metabolism via HIF1 $\alpha$ signaling under hypoxia in thyroid caner cells.

Finally, to test whether a stabilized version of HIF1 $\alpha$ can rescue NOX4 knockdown phenotypes in hypoxia regarding reduced ROS and reduced proliferation, a recombined HIF $1 \alpha$ expression vector was used from ADDGENE which used pcDNA3 as a vector backbone ${ }^{16}$. Strikingly, we found that HIF1 $\alpha$ rescued the decreased proliferation of NOX4 knockdown cells, but not restoring the mROS levels (Supplementary Fig. S4), reflecting that NOX4 regulates thyroid cancer cell growth dependent on mROS-HIF axis, rather than HIF-mROS.

p22phox is also requires for TPC-1 proliferation via mROS-HIF pathway. NADPH oxidases are differentially activated by a whole host of binding partners, including p22phox, p40phox, p47phox/NOXO1, p67phox/NOXA1 and $\mathrm{Rac}^{17}$. The NOX4 enzyme has been shown to be activated solely by the p22phox binding partner. It was demonstrated that NOX4 and p22phox expression are upregulated in thyroid cancers, linking NOX4-dependent ROS generation to cancer development or progression ${ }^{12}$. Therefore, in order to study the NOX4 activator, p22phox as with NOX4, can assist in the level of mROS in thyroid cancer cells upon hypoxia, p22phox knockout in TPC-1 cells was prepared mediated by CRISPR/Cas9 system. As shown in the immunoblot (Fig. 4A), NOX4 was positively regulated by endogenously p22phox expression, suggesting a role of p22phox in NOX4 associated signaling in thyroid cancer.

Further, we wanted to know the functional effect of p22phox on the proliferation of thyroid cancer cells in hypoxia. Cell Counting kit-8(CCK8) assays displayed that TPC-1 proliferation with p22phox knockout in hypoxia was slowed down rather than normoxia (Fig. 4B), suggesting it is functionally consistent with NOX4 in regulating the proliferation of hypoxic thyroid cancer cells. Another issue that concerned us was the regulatory relationship between P22phox and HIF1 $\alpha$, therefore, immunoblot was performed that HIF1 $\alpha$ was significantly decreased in p22phox knockout TPC-1 cells compared to the control cells in hypoxia (Fig. 4C). Consistently, the mROS level and glycolytic flux were both reduced in hypoxia once upon p22phox deletion (Fig. 4D,E). These data illustrate that p22phox regulates its functional heterodimer, NOX4, further sustain glycolytic flux via mROS-HIF $1 \alpha$ pathway in hypoxic condition.

Moreover, to understand whether add-back of NOX4 can rescue the effects of loss of p22phox, beacause they heterodimerize and this is important as p22 inhibition also results in decreased NOX4 abundance. However, the results shown that NOX4 overexpression had little effect on the mROS production, glycolytic rate and deceased cell proliferation in p22phox null cells during hypoxia (Supplementary Fig. S5), directly showing to us that NOX4 regulates proliferation of papillary thyroid carcinoma cells through a way dependent on p22phox-NOX4 heterodimer complex.

\section{Discussion}

NOX family proteins are highly regulated enzymes that in recent years have been implicated in an extremely wide array of physiological and pathophysiological processes. More and more studies have shown that NOX is closely related to the development of cancer, part of them reported that inhibition of NOX activity can inhibit the growth of tumor and promote the death of cancer cells ${ }^{12,18-21}$. And so forth, increased NOX4/p22phox in cancer might be 


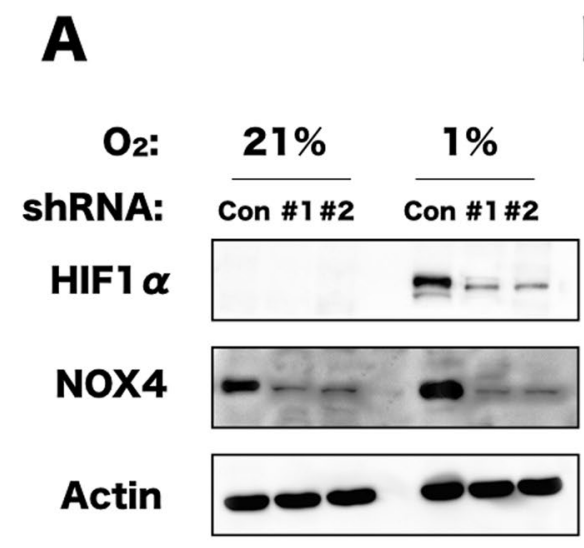

B

$1 \% \mathrm{O}_{2}$

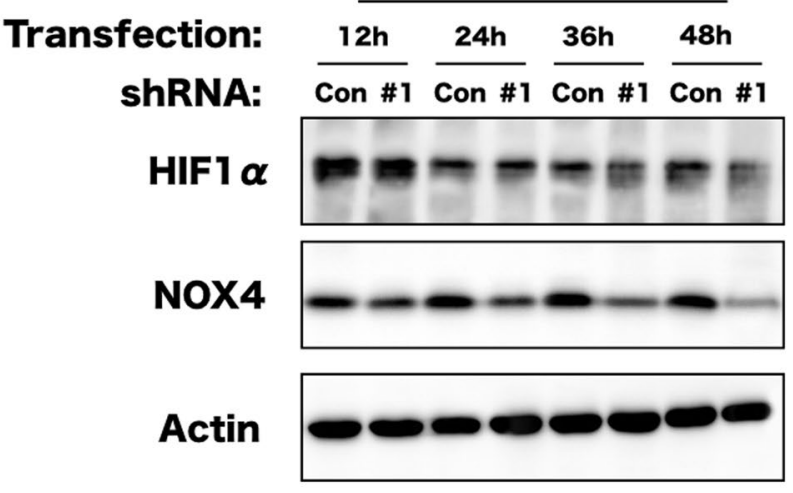

C

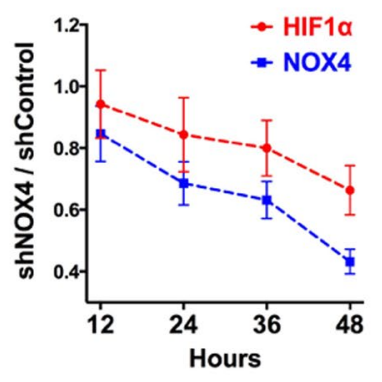

D
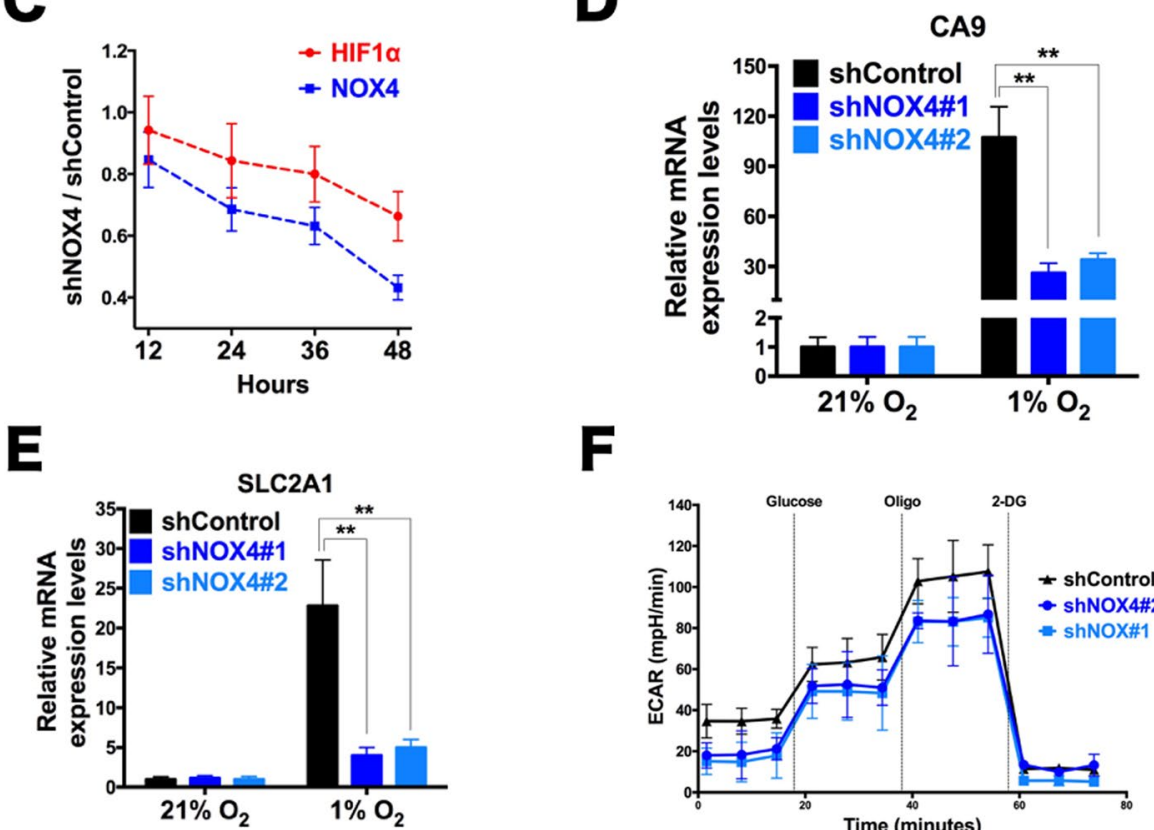

$\mathbf{F}$

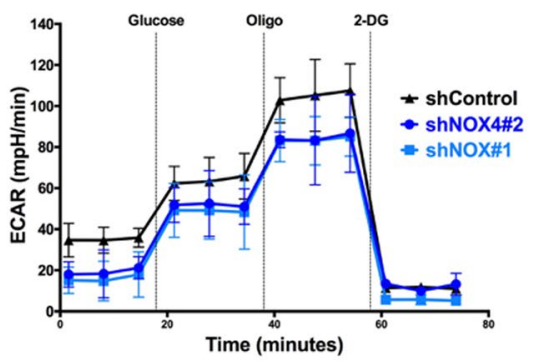

Figure 3. HIF1 $\alpha$ stabilization and glycolysis require NOX4 expression. (A) Immunoblots of NOX4 knockdown cells and its Control respectively cultured under $21 \% \mathrm{O}_{2}$ and $1 \% \mathrm{O}_{2}$ for 48 hours. (B,C) Immunoblot of TPC-1 cells grown in 1\% O2 for 48 hours after tranfected with shRNA vector targeting NOX4 for the indicated time points (B). Levels of HIF1 $\alpha$ and NOX4 quantified using Image J and normalized to actin (C). (D,E) TPC-1 cells expressing shControl or shRNA against NOX4 (shNOX4\#1,\#2) were cultured in $21 \% \mathrm{O}_{2}$ and $1 \%$ $\mathrm{O}_{2}$ (48 hours), and the fold change in CA9 (D) and SLC2A1 (E) levels was measured by qRT-PCR. (F) The extracellular acidification rate (ECAR) was measured in TPC-1 cells expressing shControl or shRNA against NOX4 (shNOX4\#1,\#2) cells treated with $1 \% \mathrm{O}_{2}$ (48 hours). Data show a representative experiment (from 3 independent experiments). Error bars $\pm \mathrm{SD}(\mathrm{n}=3)$.

related to a higher proliferation rate and tumor progression. In this study we demonstrate that NOX4 regulates thyroid cancer cell proliferation both in TPC-1 cells and xenografts. Interestingly, the latter showed contradictory results, that is, the lower proliferation markers and the level of apoptosis. Previously, Nox 4 was regarded as a target in the treatment of clear cell RCC because it is essential for full HIF2 $\alpha$ expression and activity in 786-0 renal tumor cells, even in the absence of functional VHL ${ }^{18,22}$. Our study shows that NOX4 additionally controls HIF $1 \alpha$ stabilization by the mROS production. The decreased mROS in NOX4 null cells contributes to increased HIF $1 \alpha$ destabilization. Significantly, loss of NOX4 in human papillary thyroid cancer cells decreases HIF $1 \alpha$ targeting genes such as SLC2A1 and CA9, highlighting the potential importance of NOX4-mediated metabolic reprogramming in thyroid tumor. This idea is further validated by the finding that $\mathrm{p} 22$ phox represses the glycolysis via NOX4-mROS-HIF1 $\alpha$ axis in TPC-1 cells. Taken together, we provide a mechanism whereby NOX4 or p22phox functions as a tumor glycolytic regulator which is required for thyroid carcinoma proliferation.

ROS is no longer viewed as solely damaging or signaling agents. Indeed, cancers cells take advantage of ROS producted from normal cell processes in order to drive tumor growth. Thus, heightened levels of ROS, especially mROS, are used to induce pro-tumorigenic signaling ${ }^{23}$. In our study, we demonstrated that NOX4 and its 


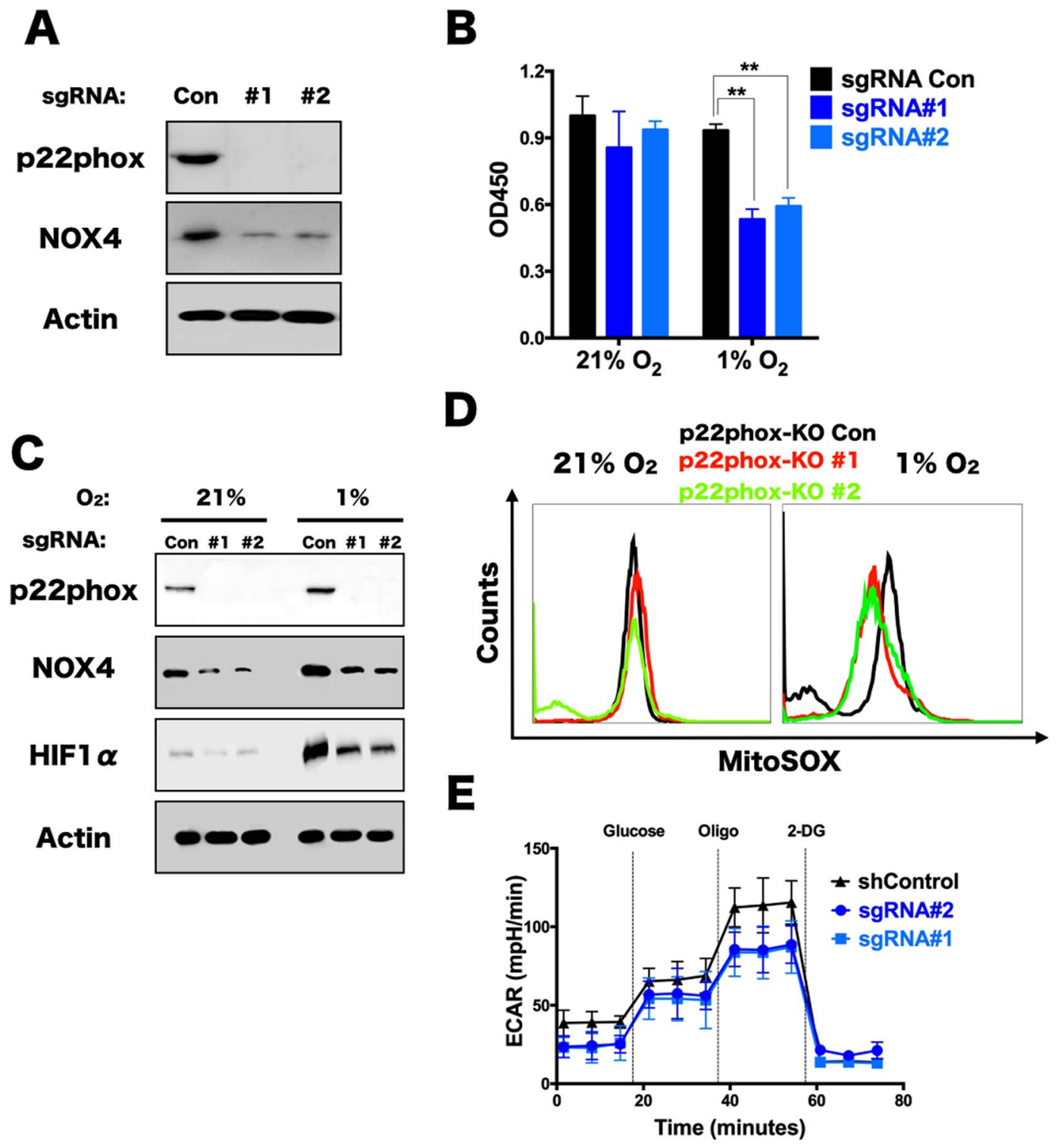

B

Figure 4. p22phox is essential for NOX4 expression and TPC-1 proliferation. (A) Immunoblots of p22phox knockout cells and its Control in TPC-1. (B) Viability assay for TPC-1 cells expressing sgRNA control(LentiCRIPSR V2.0) or sgRNA against p22phox (\#1, \#2) which were cultured in normoxia $\left(21 \% \mathrm{O}_{2}\right)$ and hypoxia $\left(1 \% \mathrm{O}_{2}\right)$ respectively for 48 hours using CCK8 assay $(n=8)$. $* * P<0.01$. (C) Immunoblots of p22phox knockout cells and its Control respectively cultured under $21 \% \mathrm{O}_{2}$ and $1 \% \mathrm{O}_{2}$ for 48 hours. (D) Mitochondrial ROS level was measured with MitoSox staining by flow cytometry analysis in TPC-1 cells expressing sgRNA control (LentiCRIPSR V2.0) or sgRNA against p22phox (\#1, \#2) cultured in $1 \% \mathrm{O}_{2}$ for 48 hours. (E) ECAR was measured in TPC-1 cells expressing expressing sgRNA control (LentiCRIPSR V2.0) or sgRNA against p22phox $(\# 1, \# 2)$ cells treated with $1 \% \mathrm{O}_{2}$ (48 hours). Data show a representative experiment (from 3 independent experiments). Error bars $\pm S D(n=3)$.

functional parter, p22phox, are specific sourse of the mROS in hypoxic microenvironment. However, we found that rescue of p22phox knockout cells with NOX4 CDS expression can not restore mROS production, which reflected that at least NOX4, must form a heterodimer with p22phox in regulating ROS production, ECAR and proliferation in PTC. Further studies should determine how much the contribution rate of NOX and p22phox protein respectively to mROS and total ROS in thyroid cancer cells exactively are, and how the sensitivity of cell response to thyroid related hormones is when NOX4 and p22phox protein levels are altered by someway.

Hypoxia is a major feature of tumor cells in vivo, as there is often an imbalance between their rapid proliferation rate and the growth of new blood vessels that supply $\mathrm{O}_{2}{ }^{24}$. In fact, in order to support growth beyond about one millimeter in diameter, tumors must recruit the expansion of new vessels to ensure proper nourishment $^{25}$. As explained previously, cells stabilize HIF under hypoxia in an mROS-dependent manner. Since mROS is known to be both necessary and sufficient for HIF stabilization under hypoxia, we report that NOX4 and p22phox in papillary thyroid carcinoma are necessary for mROS production during hypoxia. On this basis, the 
other mitochondrial function is consequently altered, such as mitochondrial membrane potential and oxygen consumption, both of which represents the basic ability level of mitochondrial. The findings suggest that NOX4 has the function of regulating the basic state of mitochondria, which may be one of the reasons why the proliferation of thyroid cancer cells slows down.

At the current state of data, we provide the first evidence that NOX4 functions as a glycolytic regulator coupling the metabolism to the papillary thyroid carcinoma proliferation. In the future, we further observe the effect of the NOX4 as a molecular target for thyroid cancer from the perspective of metabolism.

\section{Materials and Methods}

Cells and Cell Counting Kit-8. The human papillary thyroid carcinoma cell lines TPC-1 cell line(ATCC) was cultured in RPMI 1640 medium(Thermo fisher, 11875168) supplemented with $10 \%$ fetal bovine serum(Thermo fisher, 10437-028) and antibiotics in a $37^{\circ} \mathrm{C}$ incubator with $5 \% \mathrm{CO}_{2}$. The papillary thyroid cancer cell line BCPAP and K1 was obtained from Jiehui Technology (YunNan Province), and maintained in DMEM containing $10 \%$ fetal bovine serum, $100 \mathrm{U} \mathrm{ml}^{-1}$ penicillin and $100 \mathrm{U} \mathrm{ml}^{-1}$ streptomycin under a humid atmosphere of $5 \%(\mathrm{v} / \mathrm{v}) \mathrm{CO}_{2}$ at $37^{\circ} \mathrm{C}$.

Cell viability was determined by Cell Counting-Kit 8 assay (MCE). The assay was performed in 96 -well plates and the absorbance was measured at $450 \mathrm{~nm}$ in a microplate reader (Thermo Varioskan Flash) after 4 hours of incubation. The assays were performed with $\mathrm{N}=8$ and repeated 3 times.

Xenografts. For xenografts, $5 \times 10^{6} \mathrm{TPC}-1$ cells of shNOX4 and shControl stable single-cell knockdown clones were injected subcutaneously into nude mice ( 6 to 9 weeks-old males, Charles river). There were 5 mice in each group of which was injected with shControl cells and two shNOX4 cells. Tumor size was measured every 3 days, and tumors were dissected and weighed after 21 days. The average tumor volume was calculated as Volume $=\mathrm{L} \times \mathrm{W}^{2} \times 0.52$.

Tumors were fixed in $4 \%$ paraformaldehyde and embedded in paraffin. Sections were stained with hematoxylin and eosin (H\&E) in accordance with standard procedures. Immunohistochemistry was performed using tunel staining(Beyotime, C1091) and Ki67 antibody (abcam, ab833) according to manufacturer instructions.

Generation of shNOX4 stable single-cell knockdown clones. Lentiviral transduction particles for vector control or human shNOX4 were used to generate stable knockdown clones in TPC-1 cells. Lentiviral transduction particles (Sigma Aldrich product\#: TRCN0000046089 and TRCN0000046090) was used with the infected enhancer polybrene(Sigma). TPC-1 cells were infected with Lentiviral shNOX4 or shControl as per the manufacturer's instructions. 48 hours post infection, 5000 cells were selected and passed into 96-well plates in puromycin $(2000 \mathrm{ng} / \mathrm{ml})$ antibiotic for selection and maintained in puromycin $(1000 \mathrm{ng} / \mathrm{ml})$.

CRISPR/Cas9 mediated p22phox knockout clones. CRISPR/Cas9 system was used to disrupt the endogenous $\mathrm{p} 22$ phox in TPC-1 cells. The annealed double-stranded single-guide RNAs (sgRNAs) downstream of the p22phox start codon were cloned into LentiCRISPRv2 (Addgene) by BsmBI(NEB). Two sgRNAs targeting human p22phox (sgRNA\#1 5'-AACGAACAGGCGCTGGCGTC-3' and sgRNA\#2 $5^{\prime}$-GGCCATGTGGGCCAACGAAC-3') were effective to produce mutation of respective gene in genomic DNA. The reconstituted vector transfected into 293T cells using lipofectamine 3000 (Invitrogen) along with pMD2.G and psPAX2 packaging vectors to produce p22phox KO control(sgRNA Control), p22phox KO\#1 (sgRNA \#1)and p22phox KO\#2(sgRNA \#2) lentivirus. TPC-1 cells were infected with these Lentiviral and 48 hours post infection, 5000 cells were selected and passed into 96 -well plates in puromycin $(2000 \mathrm{ng} / \mathrm{ml})$ antibiotic for selection and maintained in puromycin $(1000 \mathrm{ng} / \mathrm{ml})$.

Western blotting. Cells were harvested and lysed with RIPA (20 mM Tris PH7.5, $150 \mathrm{mM} \mathrm{NaCl}, 1 \%$ Triton $\mathrm{X}-100,2.5 \mathrm{mM}$ sodium pyrophosphate, $1 \mathrm{mM}$ EDTA, $1 \% \mathrm{Na} 3 \mathrm{VO} 4,0.5 \mu \mathrm{g} / \mathrm{ml}$ leupeptin, $1 \mathrm{mM}$ PMSF). For immunoblotting, proteins from whole-cell lysates were resolved by 10 or $12 \%$ sodium dodecyl sulfate-polyacrylamide gel electrophoresis (SDS-PAGE) and then transferred to PVDF membranes. Anti-NOX4(Novus), anti-p22phox(Santa Cruz), anti-HIF1 $\alpha$ (BD science), anti-Actin(Beyotime), anti-PCNA(CST) and anti-caspase 3(abcam) antibodies were used at 1:1000 and secondary antibodies (Beyotime) conjugated with horseradish peroxidase (HRP) were used at 1:1000 dilutions in 5\% non-fat dry milk. After the final washing, PVDF membranes were exposed for an enhanced chemiluminescence assay using the Gel imaging instrument (BIO-RAD ChemiDoc MP).

qPCR. Total RNA was extracted using the TRIZOL (Invitrogen) following manufacturer's instructions and then reversed transcribed using reverse transcriptase (Takara). Quantitive PCR was performed on the BIO-RAD CFX 96 system using SYBR Green Master mix (Takara). Reactions were performed with $125 \mathrm{ng}$ of template cDNA. Transcript levels of genes were normalized to a reference index of housekeeping genes (ACTB). Primers for ACTB were used as an internal control. The primers used were as follow. NOX4: 5'-GAAAA CCCGG CTCTG GGTAG-3' (forward), 5'-TGATC CTCGG AGGTA AGCCA-3' (reverse); PCNA: 5'-GGATA CCTTGG CGCTA GTATT T- $3^{\prime}$ (forward), 5'-CACAG CTGTA CTCCT GTTCT G-3' (reverse); CA9: $5^{\prime}$-TCAGC CGCTA CTTCC AATAT G-3' (forward), 5'-TCAGC ATCAC TGTCT GGTTA AA-3' (reverse); VEGFA: 5'-CAGGA CATTG CTGTG CTTTG-3' (forward), 5' -CTCAG AAGCA GGTGA GAGTA AG-3' (reverse); ACTB: 5'-GGACC TGACT GACTA CCTCA T-3' (forward), 5'-CGTAG CACAG CTTCT CCTTA AT-3' (reverse).

Measurement of Mitochondrial ROS and potential. mROS production in trypsinized TPC-1 cells was measured using the mitoSOX(Molecular Probes, 36008) and TMRE cell fluorescent dye (50 nM) as supplier's instructions. Briefly, Apply $1.0 \mathrm{~mL}$ of $5 \mu \mathrm{M}$ MitoSOX to cover TPC- 1 cells, and incubate cells for 10 minutes at $37^{\circ} \mathrm{C}$, protected from light. Wash cells gently three times with PBS and detected by Flow Cytometry at excitation/ 
emission maxima of $510 / 580 \mathrm{~nm}$. Mitochondrial membrane potential was evaluated by fluorescence of the potential dependent TMRE probe(Thermo fisher, T668). Briefly, tripsinized cells were incubated with $50 \mathrm{nM}$ TMRE for $15 \mathrm{~min}$ in the dark, after which the media was replaced and the fluorescence was measure by flow cytometry. Cells were collected and suspended in PBS and analyzed by flow cytometer (BD FACSCalibur) and the data were analyzed using FlowJo 10.0.7 software.

OCR and ECAR Measurement. Oxygen consumption rate(OCR) and extracellular acidification rate (ECAR) were measured using a Seahorse XF24 analyzer (Seahorse Bioscience). Briefly, Oxygen consumption rate (OCR) and extracellular acidification rate (ECAR) were determined using the MitoStress kit (Seahorse Biosciences) and Glycolysis Stress kit (Seahorse Biosciences) according to the manufacturer's standard protocol. OCR and ECAR were calculated and recorded by the Seahorse XF24 software. All assays were performed with $\mathrm{N}=3$ or more per condition and repeated 3 times.

Statistical analysis. Student's t-test was used in all cellular experiments and the results from three independent experiments are presented as mean SEM. In addition, all the mRNA expression data represent the mean values with standard error of the mean (SEM). The differences in mRNA expression data were compared using the ratio t-test. All statistical analyses were performed using the GraphPad Prism software. Asterisks denote statistical significance as follows: NS, $\mathrm{P}>0.05 ; * \mathrm{P} \leq 0.05 ; * * \mathrm{P} \leq 0.01$.

Statement. We confirm that all methods were carried out in accordance with relevant guidelines and regulations of the Municipal Committee for health and family planning of Mianyang, China. We confirm that all experimental protocols in this study were approved by the Third Hospital of Mianyang (Sichuan mental health center), China.

\section{References}

1. Al-Brahim, N. \& Asa, S. L. Papillary thyroid carcinoma: an overview. Arch Pathol Lab Med 130, 1057-1062 (2006).

2. Chen, W. et al. Cancer statistics in China, 2015. CA Cancer J Clin 66, 115-132 (2016).

3. Carvalho, D. P. \& Dupuy, C. Role of the NADPH Oxidases DUOX and NOX4 in Thyroid Oxidative Stress. Eur Thyroid J 2, 160-167 (2013).

4. Bjorkman, U. \& Ekholm, R. Generation of H2O2 in isolated porcine thyroid follicles. Endocrinology 115, 392-398 (1984).

5. Maier, J. et al. Deoxyribonucleic acid damage and spontaneous mutagenesis in the thyroid gland of rats and mice. Endocrinology 147, 3391-3397 (2006).

6. Montezano, A. C. \& Touyz, R. M. Reactive oxygen species, vascular Noxs, and hypertension: focus on translational and clinical research. Antioxid Redox Signal 20, 164-182 (2014).

7. Bevilacqua, E., Gomes, S. Z., Lorenzon, A. R., Hoshida, M. S. \& Amarante-Paffaro, A. M. NADPH oxidase as an important source of reactive oxygen species at the mouse maternal-fetal interface: putative biological roles. Reprod Biomed Online 25, 31-43 (2012).

8. Ameziane-El-Hassani, R., Schlumberger, M. \& Dupuy, C. NADPH oxidases: new actors in thyroid cancer? Nat Rev Endocrinol 12, 485-494 (2016)

9. von Lohneysen, K., Noack, D., Wood, M. R., Friedman, J. S. \& Knaus, U. G. Structural insights into Nox4 and Nox2: motifs involved in function and cellular localization. Mol Cell Biol 30, 961-975 (2010).

10. Zheng, J. Energy metabolism of cancer: Glycolysis versus oxidative phosphorylation (Review). Oncol Lett 4, 1151-1157 (2012).

11. Majmundar, A. J., Wong, W. J. \& Simon, M. C. Hypoxia-inducible factors and the response to hypoxic stress. Mol Cell 40, 294-309 (2010).

12. Weyemi, U. et al. Intracellular expression of reactive oxygen species-generating NADPH oxidase NOX4 in normal and cancer thyroid tissues. Endocr Relat Cancer 17, 27-37 (2010).

13. Chandel, N. S. et al. Reactive oxygen species generated at mitochondrial complex III stabilize hypoxia-inducible factor-1alpha during hypoxia: a mechanism of O2 sensing. J Biol Chem 275, 25130-25138 (2000).

14. Schumacker, P. T. Hypoxia-inducible factor-1 (HIF-1). Crit Care Med 33, S423-425 (2005).

15. Finley, L. W. et al. SIRT3 opposes reprogramming of cancer cell metabolism through HIF1alpha destabilization. Cancer Cell 19, 416-428 (2011).

16. Yan, Q., Bartz, S., Mao, M., Li, L. \& Kaelin, W. G. Jr. The hypoxia-inducible factor 2alpha N-terminal and C-terminal transactivation domains cooperate to promote renal tumorigenesis in vivo. Mol Cell Biol 27, 2092-2102 (2007).

17. Ray, R. \& Shah, A. M. NADPH oxidase and endothelial cell function. Clin Sci (Lond) 109, 217-226 (2005).

18. Maranchie, J. K. \& Zhan, Y. Nox4 is critical for hypoxia-inducible factor 2-alpha transcriptional activity in von Hippel-Lindaudeficient renal cell carcinoma. Cancer Res 65, 9190-9193 (2005).

19. Graham, K. A. et al. NADPH oxidase 4 is an oncoprotein localized to mitochondria. Cancer Biol Ther 10, 223-231 (2010).

20. Azouzi, N. et al. NADPH Oxidase NOX4 Is a Critical Mediator of BRAF(V600E)-Induced Downregulation of the Sodium/Iodide Symporter in Papillary Thyroid Carcinomas. Antioxid Redox Signal 26, 864-877 (2017).

21. Thomasz, L. et al. Juvenal, Regulation of NADPH oxidase NOX4 by delta iodolactone (IL-delta) in thyroid cancer cells, Mol Cell Endocrinol (2017).

22. Gregg, J. L. et al. NADPH oxidase NOX4 supports renal tumorigenesis by promoting the expression and nuclear accumulation of HIF2alpha. Cancer Res 74, 3501-3511 (2014).

23. Sullivan, L. B. \& Chandel, N. S. Mitochondrial reactive oxygen species and cancer. Cancer Metab 2, 17 (2014).

24. Diebold, L., Chandel, N. S. \& Mitochondrial ROS regulation of proliferating cells. Free Radic Biol Med 100, 86-93 (2016).

25. Folkman, J. Tumor angiogenesis: therapeutic implications. N Engl J Med 285, 1182-1186 (1971).

\section{Acknowledgements}

This project was supported by the grant from the Health and family planning committee of Sichuan province, China (16PJ181, 16).

\section{Author Contributions}

Jian-feng Sheng conceived the initial concept and designed the study. Ping Tang, Hao Dang, Jie Huang, Tao Xu, Ping Yuan and Jun Hu carried out the experiments. Jie Huang offered critical opinion to improve the design and study. Jian-feng Sheng. and Ping Tang wrote the manuscript. 


\section{Additional Information}

Supplementary information accompanies this paper at https://doi.org/10.1038/s41598-018-34154-8.

Competing Interests: The authors declare no competing interests.

Publisher's note: Springer Nature remains neutral with regard to jurisdictional claims in published maps and institutional affiliations.

(c) (1) Open Access This article is licensed under a Creative Commons Attribution 4.0 International License, which permits use, sharing, adaptation, distribution and reproduction in any medium or format, as long as you give appropriate credit to the original author(s) and the source, provide a link to the Creative Commons license, and indicate if changes were made. The images or other third party material in this article are included in the article's Creative Commons license, unless indicated otherwise in a credit line to the material. If material is not included in the article's Creative Commons license and your intended use is not permitted by statutory regulation or exceeds the permitted use, you will need to obtain permission directly from the copyright holder. To view a copy of this license, visit http://creativecommons.org/licenses/by/4.0/.

(C) The Author(s) 2018 